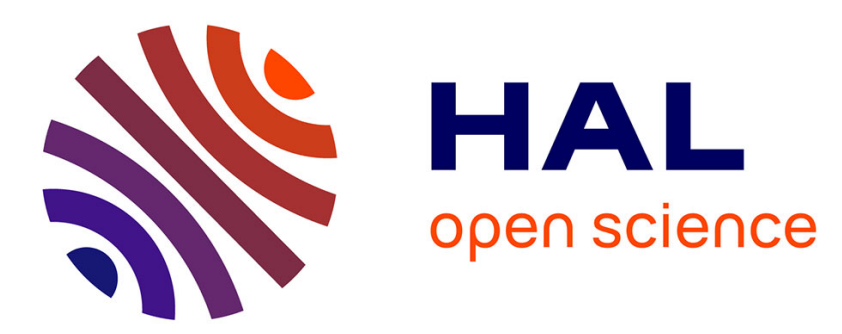

\title{
Spatial and Temporal Adaptive FIR Clutter Filtering
} Vincent Perrot, Jørgen Avdal, Lasse Løvstakken, Sébastien Salles, Didier Vray, Herve Liebgott, Ingvild Kinn Ekroll

\section{To cite this version:}

Vincent Perrot, Jørgen Avdal, Lasse Løvstakken, Sébastien Salles, Didier Vray, et al.. Spatial and Temporal Adaptive FIR Clutter Filtering. 2018 IEEE International Ultrasonics Symposium (IUS), Oct 2018, Kobe, Japan. pp.1-4, 10.1109/ULTSYM.2018.8579766 hal-02063569

\section{HAL Id: hal-02063569 https://hal.science/hal-02063569}

Submitted on 11 Mar 2019

HAL is a multi-disciplinary open access archive for the deposit and dissemination of scientific research documents, whether they are published or not. The documents may come from teaching and research institutions in France or abroad, or from public or private research centers.
L'archive ouverte pluridisciplinaire HAL, est destinée au dépôt et à la diffusion de documents scientifiques de niveau recherche, publiés ou non, émanant des établissements d'enseignement et de recherche français ou étrangers, des laboratoires publics ou privés. 


\title{
Spatial and Temporal Adaptive FIR Clutter Filtering
}

\author{
Vincent Perrot*, Jørgen Avdal ${ }^{\dagger}$, Lasse Løvstakken ${ }^{\dagger}$, Sébastien Salles ${ }^{\dagger}$, Didier Vray*, Hervé Liebgott*, \\ Ingvild Kinn Ekroll ${ }^{\dagger \ddagger}$ \\ *Univ. Lyon, INSA-Lyon, UCBL, UJM-Saint-Étienne, CNRS, Inserm, CREATIS UMR 5220, U1206, Lyon, France \\ vincent.perrot@creatis.insa-lyon.fr \\ ${ }^{\dagger}$ CIUS and Department of Circulation and Medical Imaging, Norwegian University of Science and Technology, \\ Trondheim, Norway \\ ${ }_{\ddagger}^{\ddagger}$ St. Olavs Hospital HF, Kirurgisk klinikk
}

\begin{abstract}
In this study, a new spatial and temporal FIR clutter filter has been developed using the tissue velocity. This filter uses the PSF attenuation and the tissue velocity estimates to design a filter which is time-dependent but also a function of the position relative to the tissue. The spatial and temporal adaptive FIR filter is compared with three standard FIR clutter filters: (i) a low cutoff FIR filter, (ii) a high cutoff FIR filter, and (iii) a temporal adaptive FIR filter. In the following, this comparison was made with an in vivo carotid data set from a high frame rate acquisition using unsteered plane waves. The axial component of tissue velocities was estimated using a 1D phase-based estimator. For blood flow, 2D vector velocities were estimated using a $2 \mathrm{D}$ phase-based estimator after the introduction of transverse oscillation in post-acquisition. Overall, both adaptive filters outperform the non-adaptive filters. Indeed, standard filtering methods yield strong outliers in the presence of low flow velocity for the maximum filter and high flow velocity for the minimum filter. Because the spatial and temporal adaptive filter takes into account the local tissue velocity, it facilitates flow velocity estimation closer to the wall, particularly for low flow. Indeed, only the relevant (based on the PSF) tissue velocity is removed from a specific location in the lumen. Consequently, a local high tissue velocity does not influence the cutoff velocities in the entire vessel. These new adaptive filtering methods could lead to better separation between blood and tissue in recordings from pathological carotids with spatially varying tissue velocity.

Index Terms-Adaptive, FIR, clutter filtering, tissue motion estimation, flow estimation, transverse oscillation
\end{abstract}

\section{INTRODUCTION}

Clutter filtering is an essential step in flow estimation. Its role is to remove clutter from the lumen which is, without the use of contrast agent, typically $40-60 \mathrm{~dB}$ stronger than the blood signal. Using a too high cutoff leads to suppression of low-velocity blood signal. Concerning the effect on the estimates, a high cutoff makes it challenging to extract low-velocity flow which can occur close to the wall, during diastole or in case of pathology. However, using a low cutoff leads to residual clutter in the signal. The low cutoff induces biases and outliers in the estimates because of the remaining

This work was partially funded thanks to the GdR ISIS. The Verasonics system was co-funded by the FEDER program, Saint-Etienne Metropole (SME) and Conseil General de la Loire (CG42) within the SonoCardioProtection Project headed by Prof. Pierre Croisille and Dr. Magalie Viallon, principal investigators. This work has been performed within the framework of the LABEX CELYA (ANR-10-LABX-0060) and LABEX PRIMES (ANR-10-LABX-0063) of Université de Lyon, within the program "Investissements d'Avenir" (ANR-11-IDEX-0007) operated by the French National Research Agency (ANR). clutter. Consequently, a clutter filter with a stopband frequency following the actual tissue velocity yields a lower bias and a better flow estimate. Moreover, because the tissue velocity changes during the cardiac cycle, filters with a temporal adaptive cutoff velocity can achieve better performance than the non-adaptive filters [1].

Temporal variation of the tissue surrounding the vessel is a well-known property inherent to the cardiac cycle and the pulsatility of the vessel due to the blood circulation. However, spatial variations of the tissue velocity can also occur and are due to various phenomena such as pulse wave [2], aging [3] or plaques [4] in case of a pathologic artery. Because of these spatial variations, the real tissue velocity and optimal clutter stopband frequency are not only time-dependent but also a function of the position relative to the tissue. Consequently, a clutter filter taking into account the spatial variation of the tissue velocity can outperform the standard clutter FIR concerning clutter rejection.

Various types of clutter filters have been developed since the very beginning of flow estimation and Doppler technique developments. Standard FIR filtering may by implemented as a convolution in the time-domain with the impulse response, but a relatively high filter order is needed to obtain sufficient performance [5], [6]. IIR filters can also be used and have the advantage to yield the same performance as the FIR filters with a lower order, but need to be well-initialized to guarantee stable filters [5], [6]. Both filtering methods are set with a frequency/velocity cutoff. Eigen-based filters are another possibility for separating clutter and blood signal, but they require some hypothesis or analysis to select a power threshold for the tissue signal [7]. Regression filters have also been proposed for subtraction of the clutter signal using a polynomial or linear fitting, this method requires the selection of a polynomial basis and the order of the polynomial [5], [6].

The following study will focus on FIR filters, because they are always stable and because the selected cutoff is directly corresponding to tissue velocities. This work aims to present, study and show the effect of a spatial and temporal adaptive FIR clutter filter based on the tissue velocity, using in vivo carotid data set, and with comparison with the standard FIR clutter filter as references. In section II, the material used for the recordings and the filters are presented. In section III, some 
TABLE I

ACQUISITION PARAMETERS

\begin{tabular}{ll}
\hline Parameter & Value \\
\hline Pitch & $298 \mu \mathrm{m}$ \\
Number of elements & 128 \\
Transmit frequency & $5 \mathrm{MHz}$ \\
Sampling frequency & $20 \mathrm{MHz}$ \\
Transmit pulse & $3-$ cycle sinusoidal pulse \\
Speed of sound & $1540 \mathrm{~m} / \mathrm{s}$ \\
Pulse repetition frequency & $5000 \mathrm{~Hz}$ \\
Compounding & No \\
Steering angle & $0^{\circ}$ (unsteered) \\
Transmit apodization & Tukey $50 \%$ \\
Receive apodization & Rectangular \\
Duration & $2 \mathrm{~s}$ \\
\hline \hline
\end{tabular}

results are presented obtained with the different FIR clutter filters. In section IV, a discussion and conclusion part points out the possible applications and improvements of this new type of FIR clutter filter.

\section{MATERIAL AND METHODS}

\section{A. Material}

In this study, a Verasonics ultrasound system (Verasonics Inc., Redmond, WA) with the L7-4 probe were used. Acquisition parameters are described in Table I and data were beamformed using a classic delay-and-sum (DAS) algorithm with an f-number of 1.5 .

Acquisitions were performed on healthy volunteers, six subjects under the age of thirty who stood at rest 20 minutes before the acquisition. This procedure was performed in order to ensure that all phenomena which occur during acquisition are entirely natural. The carotid presented in this study is from the volunteer with the highest estimated wall velocity with a maximum value of approximately $18 \mathrm{~mm} / \mathrm{s}$.

\section{B. Tissue motion and flow velocity estimation}

Before clutter filtering, axial tissue velocities were estimated for FIR design. At this end, axial velocities were extracted using the complex autocorrelation function [8] after I/Q demodulation at the transmit frequency

$$
v_{z}=-\frac{c \times P R F}{4 \pi f_{0}} \times \frac{\angle \hat{R}(1)}{\pi}
$$

where $c$ is the speed of sound $(\mathrm{m} / \mathrm{s}), P R F$ is the pulse repetition frequency $(\mathrm{Hz}), f_{0}$ is the transmit frequency $(\mathrm{Hz})$, and $\hat{R}(1)$ is the complex autocorrelation function at lag 1 . A spatial averaging filter with a 2D Hanning kernel of $1 \mathrm{~mm} \times 2.5 \mathrm{~mm}$ (axial $\times$ lateral) was applied to the complex autocorrelation function with an ensemble length of 16 frames to reduce variance.

Concerning the flow velocity estimation, transverse oscillation [9], [10] was introduced in post-acquisition by filtering in the Fourier domain with a Gaussian mask at the wavelength of $0.8 \mathrm{~mm}$ with a full-width at half-maximum of $2.5 \mathrm{~mm}$. After filtering, the resulting spatial Fourier spectrum is composed of four spots representing the axial (natural) and lateral

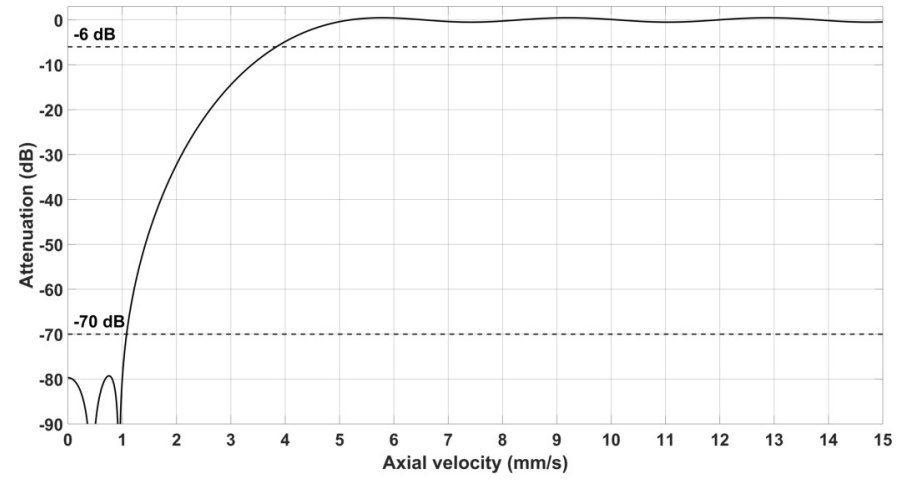

Fig. 1. Frequency response of the FIR with the lowest cutoff, all the FIR filters share the same characteristics except for the stopband frequency.

(introduced with the mask multiplication) frequencies. Then, 2D analytic signals were extracted in the Fourier domain by selecting two different single quadrants [11]. Phase shifts were estimated using the $2 \mathrm{D}$ analytics signals

$$
\left\{\begin{array}{l}
\Phi_{1}=\angle \hat{R}_{1}(1) \\
\Phi_{2}=\angle \hat{R}_{2}(1)
\end{array}\right.
$$

where $\hat{R}_{1}(1)$ is the complex autocorrelation function of the first quadrant analytics signals at lag 1 and $\hat{R}_{2}(1)$ is the same function but for the second quadrant analytic signal. Motion is recovered by projecting the phase shift onto the axial and lateral axes

$$
\left\{\begin{array}{l}
v_{z}=-\frac{c \times P R F}{4 \pi f_{0}} \times \frac{\Phi_{1}+\Phi 2}{\pi} \\
v_{x}=-\frac{c \times P R F}{4 \pi f_{x}} \times \frac{\Phi_{1}-\Phi 2}{\pi}
\end{array}\right.
$$

where $f_{x}\left(\mathrm{~m}^{-1}\right)$ is the lateral frequency of TO. A spatial average with a 2D rectangular kernel $1 \mathrm{~mm} \times 2.5 \mathrm{~mm}$ (axial $\times$ lateral) was applied to the complex autocorrelation function with an ensemble length of 64 frames to reduce variance.

\section{FIR design}

1) FIR characteristics: All the FIR filters used in this study have the same properties except for the stopband frequency. The order has been set to 200 , the attenuation to $-70 \mathrm{~dB}$, the ripple to $3 \mathrm{~dB}$, the design method was equiripple, and the cutoff frequencies were set to 2 times the corresponding tissue velocity. An example of the frequency response of an FIR clutter filter (minimum) is shown in Fig. 1.

2) Minimum filter: The minimum FIR clutter filter corresponds to the minimum wall velocity during all the cardiac cycles. For this data set, the corresponding cutoff velocity is around $1 \mathrm{~mm} / \mathrm{s}$ and this cutoff is shown in Fig. 2 with the other standard cutoffs.

3) Maximum filter: The maximum FIR clutter filter has a cutoff set on the maximum estimated tissue velocity estimated in all the frames. The corresponding value is approximately $37 \mathrm{~mm} / \mathrm{s}$ and is shown in Fig. 2 with the other standard cutoffs. 


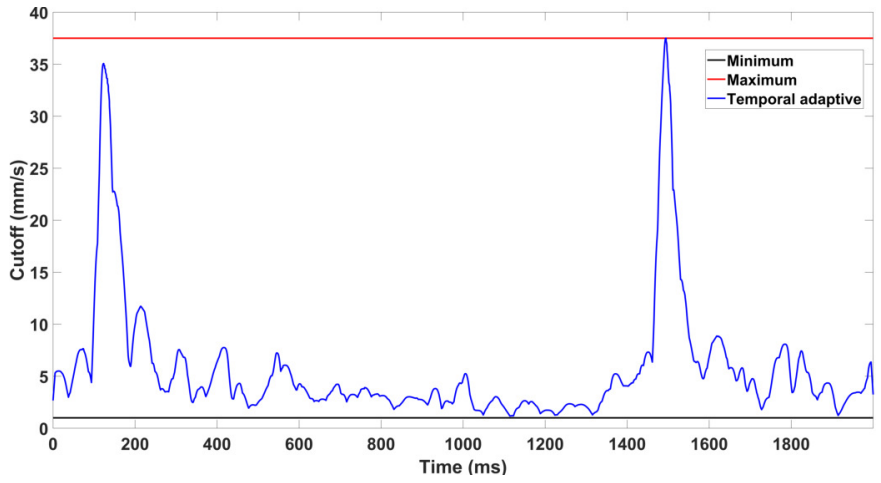

Fig. 2. Comparison of the different cutoffs for the maximum, minimum, and temporal adaptive FIR.

4) Temporal adaptive filter: The temporal adaptive filter has a temporal cutoff based on the maximum estimated tissue velocity in the current frame. The curve is shown in Fig.2 and corresponds well to the cardiac cycle. The two peaks at $150 \mathrm{~ms}$ and $1500 \mathrm{~ms}$ correspond to strong expansions of the wall at the beginning of systole.

5) Spatial and temporal adaptive filter: The spatial and temporal adaptive filter is based on two characteristics: $(i)$ the tissue velocity extracted from the data and (ii) the beamformed PSF measured on a $30 \mu \mathrm{m}$ chirurgical wire using the same insonated sequence. The stopband frequency is computed using the following procedure for each pixel in the data:

1) A window surrounding the pixel of interest is created based on the beamformed PSF at $-70 \mathrm{~dB}$ (corresponding to the attenuation target).

2) Each pixel in this window may influence the pixel of interest. The tissue velocities in this window are listed, and a corresponding cutoff velocity is computed for each tissue velocity in the window.

3) For each pixel in this window, a filter cutoff candidate is calculated so that the combined attenuation of the filter and PSF is $-70 \mathrm{~dB}$. For instance, if for a point the cutoff is $1 \mathrm{~mm}$ (curve example in Fig. 1) and the PSF attenuation is $-10 \mathrm{~dB}$ for this pixel, the final cutoff is around $0.8 \mathrm{~mm} / \mathrm{s}$ which corresponds to translate the template from the $1 \mathrm{~mm} / \mathrm{s}$ cutoff to a lower cutoff to obtain $-60 \mathrm{~dB}$ attenuation at the initial $1 \mathrm{~mm} / \mathrm{s}$.

4) From the list of cutoffs taking into account the PSF, the maximum stopband frequency is chosen for the pixel of interest.

5) This operation is applied for all frames and each pixel.

6) Finally, each pixel and each frame are clutter filtered using the stopband frequencies estimated from this procedure.

During this procedure, the PSF and the local tissue velocity are taken into account. This could yield better performance in case of local high tissue velocity like the example in Fig. 3; in this example at the very beginning of the systole, the proximal wall velocity is higher than the distal wall velocity, and the maximum proximal wall velocity is localized at the

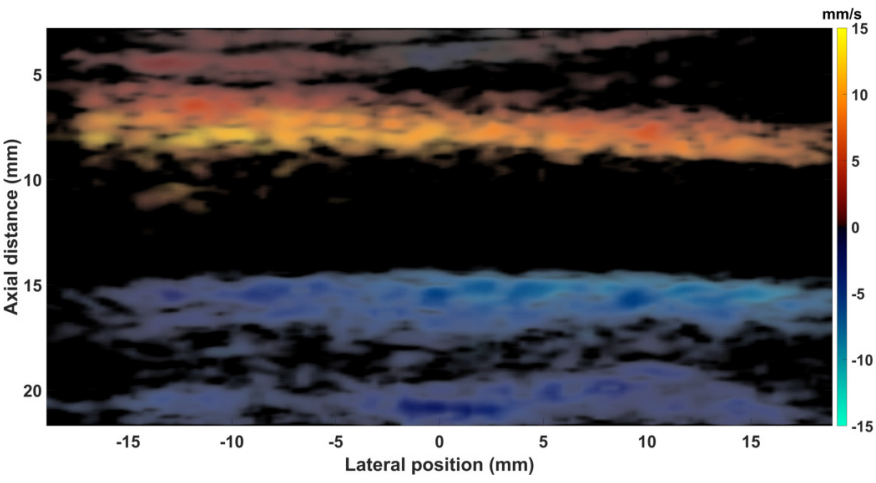

Fig. 3. B-mode image superimposed with the estimated wall velocity, a positive value (red color) stands for displacement toward the probe while a negative value (blue color) corresponds to a motion away from the transducer. This frame corresponds to the moment where the wall velocity is at a maximum due to the aortic valve openings at the beginning of the systole.

left part of the carotid. This difference can be explained by the propagation of a pulse wave due to the opening of the aortic valve and by a different elastic property along the wall. Consequently, a significant variation in axial velocities can be seen along the lateral axis.

\section{RESULTS}

The clutter filters described in Section II were applied to the same carotid data set. For comparison, the temporal evolution of the flow in the center of the lumen was extracted and the temporal evolution of the flow $1 \mathrm{~mm}$ away from the proximal wall for both adaptive clutter filters (Fig. 4). Firstly, the maximum clutter filter seems to extract the flow correctly during systole (Fig. 4a, cyan curve) but fails in case of low flow velocity during diastole because the blood signal is strongly attenuated. The minimum cutoff filter has the opposite behavior. The flow during diastole is measured adequately because the wall is slow-moving, but velocities during systole are underestimated, probably because signals from the rapid wall motion are not sufficiently attenuated. The two adaptive filters allow for flow estimation during both systole and diastole in the center of the lumen. However, closer to the wall the temporal adaptive filter (Fig. 4b, black curve) generate an unexpected estimate at the very beginning of systole when the flow is minimum, but the wall starts moving. This outlier does not occur with the spatial and temporal filter (Fig. 4b, red curve). This effect may be explained using Fig. 3; indeed, at this time, the wall shows a local high velocity. For the spatial and temporal adaptive filter, the local high velocity influences only a region surrounding this area. Consequently, at this particular time point, the spatial and temporal adaptive filter seems to outperform the temporal adaptive method at the cost of a higher computation time due to the more complex work flow.

Overall, from the vector velocity fields (Fig. 5) the spatial and temporal adaptive filter (Fig. 5, bottom) seems to outperform the temporal adaptive filter (Fig. 5, top) by providing less artifacts closer to the wall. Moreover, close to the left part 


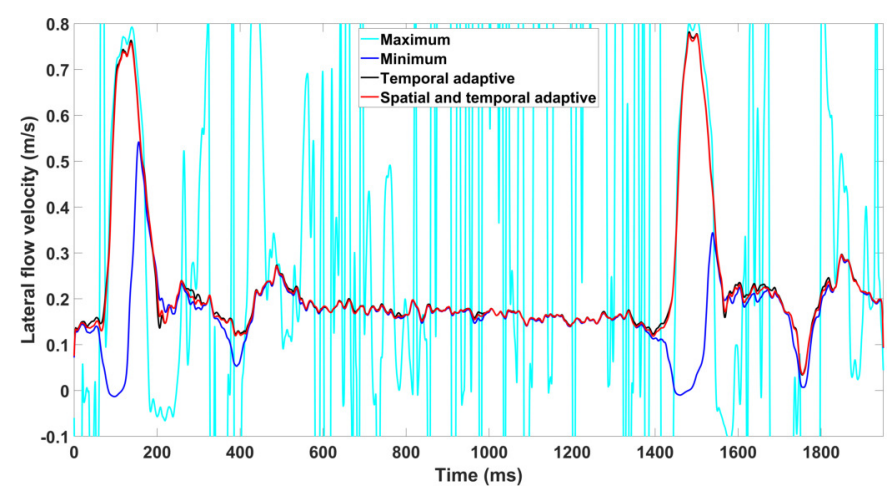

(a)

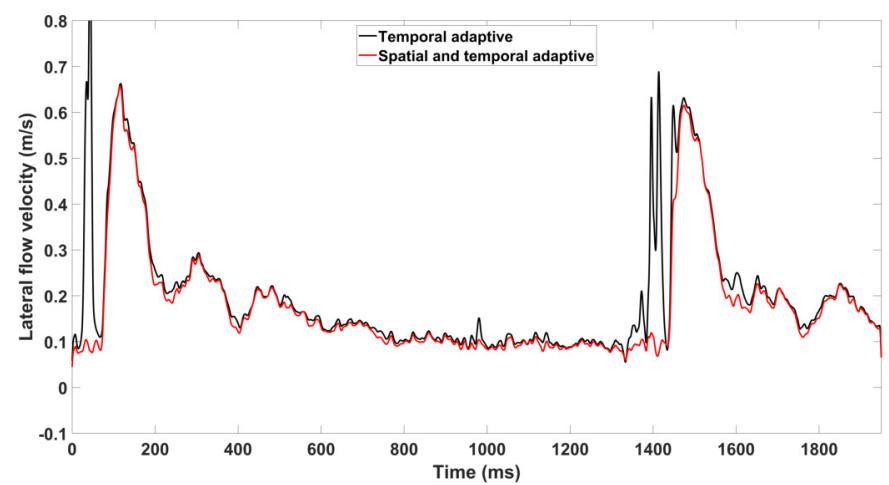

(b)

Fig. 4. (a) Temporal evolution of the flow at the center of the lumen for the minimum, maximum, temporal adaptive, and spatial and temporal adaptive FIR clutter filters. (b) The temporal evolution of the flow $1 \mathrm{~mm}$ away from the proximal wall along the central element for the temporal and spatial and temporal adaptive FIR filters

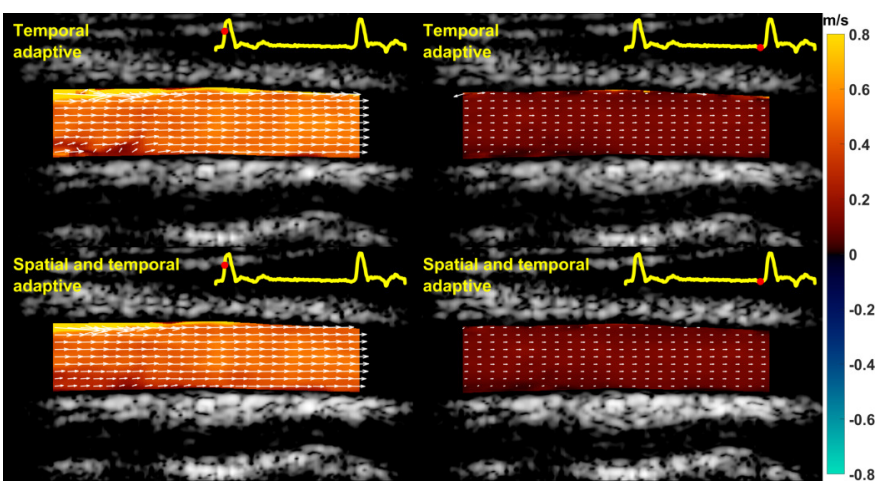

Fig. 5. Vector velocity fields for the temporal adaptive (top) and the spatial and temporal adaptive (bottom) FIR filters. Fields are shown at two different times, before the systolic peak (left) which occurs when the wall velocity is maxima and at the very end of the diastole (right) when the flow is minimal, but the wall starts moving.

of the distal wall, during systole, the flow is more laminar with the spatial and temporal adaptive filter (Fig. 5, bottom left) than the temporal adaptive filter (Fig. 5, top left).

\section{CONCLUSION AND DISCUSSION}

In this work, a spatial and temporal adaptive FIR clutter filter was presented and compared with two standard FIR clutter filters and a temporal adaptive filter, using data recorded from the carotid of a healthy volunteer. It is shown that both adaptive clutter filters outperform the non-adaptive ones. However, by taking into account the local tissue velocity and the PSF attenuation, the spatial and temporal FIR clutter filter can provide a better estimate close to the wall in case of high local tissue velocity and low flow velocity. However, this new filter is time-demanding because of the high computation cost of the complex workflow for computing the filter cutoff in each pixel at each time point.

The spatial and temporal FIR clutter filter could lead to improved velocity estimation in specific cases such as pathological carotid with plaques which may contain localized high tissue velocities. The same approach could also be extended to other filters based on frequency cutoffs.

\section{REFERENCES}

[1] I. K. Ekroll and J. Avdal, "Adaptive clutter filtering based on tissue vector velocities," in 2017 IEEE International Ultrasonics Symposium (IUS). IEEE, sep 2017.

[2] W. Nichols, M. O'Rourke, and C. Vlachopoulos, "Properties of the arterial wall: practice," in McDonald's Blood Flow in Arteries 6th Edition: Theoretical, Experimental and Clinical Principles, R. Reneman, Ed. CRC Press, jul 2011, pp. 77-110.

[3] — , "Aging," in McDonald's Blood Flow in Arteries 6th Edition: Theoretical, Experimental and Clinical Principles, R. Reneman, Ed. CRC Press, jul 2011, pp. 411-446.

[4] R. T. Lee, A. J. Grodzinsky, E. H. Frank, R. D. Kamm, and F. J. Schoen, "Structure-dependent dynamic mechanical behavior of fibrous caps from human atherosclerotic plaques." Circulation, vol. 83, no. 5, pp. 1764-1770, may 1991.

[5] H. Torp, "Clutter rejection filters in color flow imaging: a theoretical approach," IEEE Transactions on Ultrasonics, Ferroelectrics and Frequency Control, vol. 44, no. 2, pp. 417-424, mar 1997.

[6] S. Bjaerum, H. Torp, and K. Kristoffersen, "Clutter filters adapted to tissue motion in ultrasound color flow imaging," IEEE Transactions on Ultrasonics, Ferroelectrics and Frequency Control, vol. 49, no. 6, pp. 693-704, jun 2002.

[7] A. Yu and L. Lovstakken, "Eigen-based clutter filter design for ultrasound color flow imaging: a review," IEEE Transactions on Ultrasonics, Ferroelectrics and Frequency Control, vol. 57, no. 5, pp. 1096-1111, may 2010.

[8] T. Loupas, J. Powers, and R. Gill, "An axial velocity estimator for ultrasound blood flow imaging, based on a full evaluation of the doppler equation by means of a two-dimensional autocorrelation approach," IEEE Transactions on Ultrasonics, Ferroelectrics and Frequency Control, vol. 42, no. 4, pp. 672-688, jul 1995.

[9] J. Jensen and P. Munk, "A new method for estimation of velocity vectors," IEEE Transactions on Ultrasonics, Ferroelectrics and Frequency Control, vol. 45, no. 3, pp. 837-851, may 1998.

[10] H. Liebgott, A. Basarab, P. Gueth, D. Friboulet, and P. Delachartre, "Transverse oscillations for tissue motion estimation," Ultrasonics, vol. 50, no. 6, pp. 548-555, may 2010.

[11] T. Bulow and G. Sommer, "Hypercomplex signals-a novel extension of the analytic signal to the multidimensional case," IEEE Transactions on Signal Processing, vol. 49, no. 11, pp. 2844-2852, 2001. 